\title{
AN UNSTEADY HM (HYDRO-MAGNETIC) STREAM OF A STICKY LIQUID BETWEEN TWO OSCILLATING PLATES BY ELZAKI TRANSFORM
}

\author{
B. VENNILA ${ }^{1} \&$ K. SUGANTHI ${ }^{2}$ \\ ${ }^{I}$ Department of Mathematics, School of Basic Sciences, College of Engineering and Technology, SRM Institute of Science and \\ Technology, Kattankulathur, Tamil Nadu, India \\ ${ }^{2}$ Department of Electronics and Communication, School of Electrical and Electronics Engineering, College of Engineering and \\ Technology, SRM Institute of Science and Technology, Kattankulathur, Tamil Nadu, India

\begin{abstract}
This paper focuses on the movement of a thick liquid between two oscillating plates. The equipped method called, Elzaki transform method derives accurate solutions for the liquefied velocity, wall shear stress as well as skin friction. The outcomes are exposed graphically for special values of a time phase of oscillations of the plates, and tables are presented for skin friction, wall shear stress with different periods.

KEYWORDS: Unsteady Hydromagnetic Flows, Elzaki Transform, Hydromagnetic Parameter, Wall Shear Stress \& Skin Friction
\end{abstract}

Received: May 2, 2020; Accepted: May 22, 2020; Published: Jun 12, 2020; Paper Id.: IJMPERDAUG20207

\section{INTRODUCTION}

Hydromagnetic incompressible viscous flow has a lot of significant engineering applications such as magneto hydrodynamic (MHD) control maker and the cooling of reactors. But in current time, the possessions of a crossway applied magnetic field on the run of an electrically conducting viscous solution have been discussed expansively using to its astrophysical, geophysical, and business applications.

We find a number of applications in the polymer manufacturing plus metallurgy, where HM techniques are being used. The uneven HM flood of a dusty gluey fluid between two oscillating plates is examined [1]. The HM flow at an oscillating plate is investigated [2]. An HM stream of a sandy visco-elastic flowing connecting two unbounded parallel plates is developed [3]. The Time-dependent pour of HM nanofluid in a rotating cone is examined [4].

The HM flow of an Oldroyd - B watery stuck between two oscillating plates was discussed by Arun Kumar Ghosh et al [5]. The numerical solution for HM gush of a filthy fluid involving two parallel plates calculated [6]. The HM Stokes run in a revolving fluid with balanced little particles discussed. Numerical technique of Hydromagnetic stream and heat transfer of a second- grade viscoelastic liquid calculated [7]. The uneven laminar, joint forced-free convection flow in a four-sided figure opening in the occurrence of interior heat creation or amalgamation, and a magnetic field is formulated by Chamkha [8]. HM two - dimensional border level flow with heat transfer of a viscous, incompressible, electrically conducting, heat- absorbing in addition to an optically wide heat-radiating fluid over a porous exponentially stretching leaf is investigated [9].

Numerical solution for HM current due to a revolving porous floppy in a nanofluid with internal heat absorption is derived [10]. The Hall property on Hydromagnetic free convection in a heated straight up channel in 
the presence of an inclined magnetic field plus thermal energy is explained [11]. The surge of a tacky incompressible and electrically conducting liquid twisted by the harmonically oscillating partition of an inestimable area in the presence of a transverse magnetic field is explored [12]. An HM flow of a conducting micropolar fluid above a flat wall with heat transfer is studied [13]. A numerical form to study nanofluid film over an unsteady stretching sheet in the presence of Hydromagnetic flow is developed [14]. The warm and mass transport on the non-linear HM flow of a surface with inside heat generation as well as thermal stratification effects are studied [15]. Applications of new transformation Elzaki transform to partial differential equations are introduced by Tarig Elzaki et al [16]. The flows of a stratified watery from first to last the porous medium linking two oscillating saucers are discussed 17]. Unsteady free convection gush of viscous fluid due to non-coaxial rotation and fluid at perpetuity over an oscillating perpendicular plate with constant wall temperature is investigated [18]. The heat transmits analysis in mixed convection flow of Maxwell fluid above an oscillating plate is discussed [19]. Hydromagnetic oscillating convective run throughout a permeable standard in a rotating vertical porous channel with thermal radiation outcome is measured [20].

The effect of emission and hall current on an unsteady MHD free convective flow in a vertical channel with a porous medium is studied [21]. The differential geometry techniques to the flow of a viscous incompressible embedded two parallel oscillating plates are discussed [22]. The three-dimensional flow of a viscous incompressible electrically conducting fluid close to an unbounded plate of non-conductor is premeditated [23].

The unsteady MHD two- dimensional edge stratum flow of a second -grade viscoelastic fluid over an oscillatory stretching face is analyzed [24]. An unsteady Magneto Hydrodynamic free convection flow past a touching plate maintained at regular heat flux and set in a viscous fluid is offered [25].

Seeing the growth of the study in this field and its applications in various sectors, it has been chosen that the present work should be determined on the flow of viscoelastic fluid through oscillating plates under the effect of the Elzaki transform.

\section{PROBLEM DESCRIPTION}

Here, HM flow of a sticky liquid stuck between two oscillating plates is considered. The solution system is enclosed by two parallel plates at an expanse ' $h$ ' away from each other.

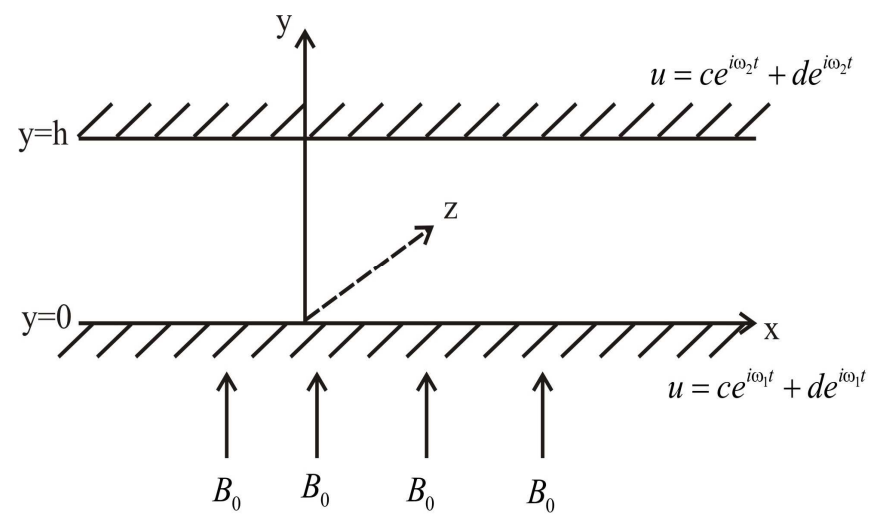

Figure 1: Flow Geometry.

Thus, the wavering action of an incompressible electrically conducting sticky fluid flow within $0 \leq y \leq h$ is generated by the equation 


$$
\frac{\partial u}{\partial t}=v \frac{\partial^{2} u}{\partial y^{2}}-n u
$$

Topic to no-slip boundary conditions at the plate are

$$
\begin{aligned}
& u=a_{11} e^{i \omega_{1} t}+b_{11} e^{-i \omega_{1} t} \text { at } \mathrm{y}=0, \mathrm{t}>0 \\
& u=c_{11} e^{i \omega_{2} t}+d_{11} e^{-i \omega_{2} t} \text { at } \mathrm{y}=\mathrm{h}, \mathrm{t}>0 \\
& \mathrm{u}=0 \text { at } t \leq 0 \text { for all } \mathrm{y} \text { in } \mathrm{h}
\end{aligned}
$$

where u represents the velocity component of the fluid, $n=\frac{\sigma_{0} B_{0}^{2}}{\rho}$ is the hydrodynamic parameter, $\sigma_{0}$ is electrical conductivity, $B_{0}$ is a magnetic field and $\rho$ is the density of the fluid, a, b, c,and d are complex constants.

We introduce the following dimensionless quantities

$$
y^{\prime}=\frac{y}{h^{\prime}}, u^{\prime}=\frac{u h}{v}, t^{\prime}=\frac{\gamma t}{h^{2}}, h^{\prime}=\frac{n h^{2}}{v},\left(\sigma_{1}, \sigma_{2}\right)=\frac{h^{2}}{v}\left(\omega_{1}, \omega_{2}\right)
$$

\section{ELZAKI TRANSFORM METHOD}

Equations (1), (2) and (3), we get,

$$
\begin{aligned}
& \frac{\partial u}{\partial t}=\frac{\partial^{2} u}{\partial y^{2}}-n u \\
& u=a_{11} e^{i \sigma_{1} t}+b_{11} e^{-i \sigma_{1} t} \text { on } \mathrm{y}=0, \mathrm{t}>0 \\
& u=c_{11} e^{i \sigma_{2} t}+d_{11} e^{-i \sigma_{2} t} \text { on } \mathrm{y}=1, \mathrm{t}>0 \\
& u=0 \text { at } t \leq 0 \text { for all } \mathrm{y}
\end{aligned}
$$

let $\bar{T}(y, v)$ be the Elzaki transform of $u(y, t)$.

(i.e.) $E[u(y, t)]=\bar{T}(y, v)$.

Taking the Elzaki transform of (6) and on the use of (9), we have

$$
\begin{aligned}
& \frac{\bar{T}(y, v)}{v}-v u(y, 0)=\frac{d^{2} \bar{T}}{d y^{2}}-n \bar{T}(y, v) \\
& \frac{d^{2} \bar{T}}{d y^{2}}-\left(n+\frac{1}{v}\right) \bar{T}(y, v)=0
\end{aligned}
$$

A solution of equation (11) is

$$
\bar{T}(y, v)=A \cosh p y+B \sinh p y
$$


Where $\bar{T}(y, v)=v \int_{0}^{\infty} e^{\frac{t}{v}} u(y, t) d t$

and $p^{2}=n+\frac{1}{v}$

Equation (13) denotes the Elzaki transform of $u(y, t)$.

Apply Elzaki transform of (7) and (8), we get

$\bar{T}(0, v)=\frac{a_{11} v^{2}}{1-i v \sigma_{1}}+\frac{b_{11} v^{2}}{1+i v \sigma_{1}}$

$\bar{T}(1, v)=\frac{c_{11} v^{2}}{1-i v \sigma_{2}}+\frac{d_{11} v^{2}}{1+i v \sigma_{2}}$

Using equations (14) and (15) in eqn. (12), we have

$\bar{T}(y, v)=\left(\frac{a_{11} v^{2}}{1-i v \sigma_{1}}+\frac{b_{11} v^{2}}{1+i v \sigma_{1}}\right) \cosh p y+\left[\left(\frac{c_{11} v^{2}}{1-i v \sigma_{2}}+\frac{d_{11} v^{2}}{1+i v \sigma_{2}}\right)-\left(\frac{a_{11} v^{2}}{1-i v \sigma_{1}}+\frac{b_{11} v^{2}}{1+i v \sigma_{1}}\right) \cosh p y\right] \frac{\sinh p y}{\sinh p}$
$\bar{T}(y, v)=v\left\{\left(\frac{a_{11}}{\frac{1}{v}-i \sigma_{1}}+\frac{b_{11}}{\frac{1}{v}+i \sigma_{1}}\right) \cosh p y+\left[\left(\frac{c_{11}}{\frac{1}{v}-i \sigma_{2}}+\frac{d_{11}}{\frac{1}{v}+i \sigma_{2}}\right)-\left(\frac{a_{11}}{\frac{1}{v}-i \sigma_{1}}+\frac{b_{11}}{\frac{1}{v}+i \sigma_{1}}\right) \cosh p\right] \frac{\sinh p y}{\sinh p}\right\}$

By duality property of Elzaki and Laplace transform

$$
\begin{aligned}
& \bar{u}(y, s)=\left(\frac{a_{11}}{s-i \sigma_{1}}+\frac{b_{11}}{s+i \sigma_{2}}\right) \cosh p y+\left[\left(\frac{c_{11}}{s-i \sigma_{2}}+\frac{d_{11}}{s+i \sigma_{2}}\right)-\left(\frac{a_{11}}{s-i \sigma_{1}}+\frac{b_{11}}{s+i \sigma_{2}}\right) \cosh p\right] \frac{\sinh p y}{\sinh p} \\
& \bar{u}(y, s)=\left(\frac{a_{11}}{s-i \sigma_{1}}+\frac{b_{11}}{s+i \sigma_{1}}\right) \frac{\sinh p(1-y)}{\sinh p}+\left(\frac{c_{11}}{s-i \sigma_{2}}+\frac{d_{11}}{s+i \sigma_{1}}\right) \frac{\sinh p y}{\sinh p} \\
& u(y, t)=L^{-1}\left\{\frac{\sinh p(1-y)}{\sinh p}\left(\frac{a_{11}}{s-i \sigma_{1}}+\frac{b_{11}}{s+i \sigma_{1}}\right)+\frac{\sinh p y}{\sinh p}\left(\frac{c_{11}}{s-i \sigma_{2}}+\frac{d_{11}}{s+i \sigma_{2}}\right)\right\}
\end{aligned}
$$

Equation (20) can be solved by the method of residues

$\frac{1}{2 \pi i} \int_{\gamma-i \infty}^{\gamma+i \infty} \frac{e^{s t} \sinh (s+n)^{1 / 2} y}{\sinh (s+n)^{1 / 2}}\left(\frac{c_{11}}{s-i \sigma_{2}}\right) d s=$ sum of the residues of the integrand at the pole

The residue at $s=i \sigma_{2}=\frac{c_{11} e^{i \sigma_{2}} \sinh \left(n+i \sigma_{2}\right)^{1 / 2} y}{\sinh \left(n+i \sigma_{2}\right)}$

The residue at $s=s_{n}=\sum_{n=1}^{\infty} \frac{2 c_{11} n \pi(-1)^{n} e^{-\left(\mathrm{n}^{2} \pi^{2}+\mathrm{n}\right) \mathrm{t}} \sin \pi y}{\mathrm{n}^{2} \pi^{2}+\mathrm{n}++i \sigma_{2}}$ 


$$
\begin{aligned}
&= a_{11}\left\{\frac{e^{i \sigma_{1} t} \sinh \left(n+i \sigma_{2}\right)^{1 / 2}(1-y)}{\sinh \left(n+i \sigma_{2}\right)}+2 \pi \sum_{n=1}^{\infty} \frac{(-1)^{n} n \sin n \pi(1-y) e^{-\left(\mathrm{n}^{2} \pi^{2}+\mathrm{n}\right) \mathrm{t}}}{\left(\mathrm{n}^{2} \pi^{2}+\mathrm{n}+i \sigma_{1}\right)}\right\} \\
&+b_{11}\left\{\frac{e^{-i \sigma_{1} t} \sinh \left(n-i \sigma_{2}\right)^{1 / 2}(1-y)}{\sinh \left(n-i \sigma_{2}\right)}+2 \pi \sum_{n=1}^{\infty} \frac{n \sin n \pi(1-y) e^{-\left(\mathrm{n}^{2} \pi^{2}+\mathrm{n}\right) \mathrm{t}}}{\left(\mathrm{n}^{2} \pi^{2}+\mathrm{n}-i \sigma_{1}\right)}\right\} \\
&+c_{11}\left\{\frac{e^{i \sigma_{2} t} \sinh \left(n+i \sigma_{2}\right)^{1 / 2} y}{\sinh \left(n+i \sigma_{2}\right)}+2 \pi \sum_{n=1}^{\infty} \frac{(-1)^{n} n \sin n \pi y e^{-\left(\mathrm{n}^{2} \pi^{2}+\mathrm{n}\right) \mathrm{t}}}{\left(\mathrm{n}^{2} \pi^{2}+\mathrm{n}+i \sigma_{2}\right)}\right\} \\
&+d_{11}\left\{\frac{e^{-i \sigma_{2} t} \sinh \left(n-i \sigma_{2}\right)^{1 / 2} y}{\sinh \left(n-i \sigma_{2}\right)}+2 \pi \sum_{n=1}^{\infty} \frac{n \sin n \pi y e^{-\left(\mathrm{n}^{2} \pi^{2}+\mathrm{n}\right) \mathrm{t}}}{\left(\mathrm{n}^{2} \pi^{2}+\mathrm{n}-i \sigma_{2}\right)}\right\} \\
&+u(y, t)=a_{11}\left\{\left(A_{1} \cos \sigma_{1} t-B_{1} \sin \sigma_{1} t\right)+i\left(B_{1} \cos \sigma_{1} t+A_{1} \sin \sigma_{1} t\right)\right\} \\
&+b_{11}\left\{\left(A_{1} \cos \sigma_{1} t-B_{1} \sin \sigma_{1} t\right)-i\left(B_{1} \cos \sigma_{1} t+A_{1} \sin \sigma_{1} t\right)\right\} \\
&+\mathrm{c}_{11}\left\{\left(A_{2} \cos \sigma_{2} t-B_{2} \sin \sigma_{2} t\right)+i\left(B_{2} \cos \sigma_{2} t+A_{2} \sin \sigma_{2} t\right)\right\} \\
&+\mathrm{d}_{11}\left\{\left(A_{2} \cos \sigma_{2} t-B_{2} \sin \sigma_{2} t\right)-i\left(B_{2} \cos \sigma_{2} t+A_{2} \sin \sigma_{2} t\right)\right\} \\
&+2 \pi \sum_{n=1}^{\infty} \mathrm{n}(-1)^{n} \sin n \pi(1-y)\left(\Delta_{1}+\Delta_{2}\right) e^{\lambda_{1} \mathrm{t}}+2 \pi \sum_{n=1}^{\infty} \mathrm{n}(-1)^{n} \sin n \pi y\left(\Delta_{3}+\Delta_{4}\right) e^{\lambda_{1} \mathrm{t}}
\end{aligned}
$$

Where

$$
A_{1}=\frac{1}{K_{3}}\left\{\mathrm{~K}_{1} \operatorname{cosa} \mathrm{a}_{2}(1-\mathrm{y}) \operatorname{sinha}_{1}(1-\mathrm{y})+\mathrm{K}_{2} \cosh \mathrm{a}_{1}(1-\mathrm{y}) \operatorname{sina}_{2}(1-\mathrm{y})\right\}
$$

$B_{1}=\frac{1}{K_{3}}\left\{\mathrm{~K}_{1} \sin \mathrm{a}_{2}(1-\mathrm{y}) \cosh \mathrm{a}_{1}(1-\mathrm{y})-\mathrm{K}_{2} \cos \mathrm{a}_{2}(1-\mathrm{y}) \sinh \mathrm{a}_{1}(1-\mathrm{y})\right\}$

$A_{2}=\frac{1}{K_{3}^{\prime}}\left\{\mathrm{K}_{1}^{\prime} \cos \mathrm{a}_{4} \mathrm{y} \sinh \mathrm{a}_{3} \mathrm{y}+\mathrm{K}_{2}^{\prime} \sin \mathrm{a}_{4} \mathrm{y} \cosh \mathrm{a}_{3} \mathrm{y}\right\}$

$B_{2}=\frac{1}{K_{3}^{\prime}}\left\{\mathrm{K}_{1}^{\prime} \sin \mathrm{a}_{4} \mathrm{y} \cosh \mathrm{a}_{3} \mathrm{y}-\mathrm{K}_{2}^{\prime} \cos \mathrm{a}_{4} \mathrm{y} \sinh \mathrm{a}_{4} \mathrm{y}\right\}$

Where $\left(a_{1}, a_{2}\right)=\left(\frac{\sqrt{n+\sigma_{1}^{2}} \mp n}{2}\right)^{1 / 2}$

$$
\left(a_{3}, a_{4}\right)=\left(\frac{\sqrt{n+\sigma_{2}^{2}} \mp n}{2}\right)^{1 / 2}
$$

Where $K_{1}=\sinh a_{1} \cos a_{2} ; K_{2}=\cosh a_{1} \sin a_{2}$

$K_{1}^{\prime}=\sinh a_{3} \cos a_{4} ; K_{2}^{\prime}=\cosh a_{3} \sin a_{4}$

$K_{3}=K_{1}^{2}+K_{2}^{2} ; K_{3}^{\prime}=K_{1}^{\prime 2}+K_{2}^{\prime 2}$

$$
\Delta_{1}=\left(\frac{a_{11}}{\lambda_{1}+i \sigma_{1}}\right) ; \Delta_{2}=\left(\frac{b_{11}}{\lambda_{1}-i \sigma_{1}}\right)
$$


$\Delta_{3}=\left(\frac{c_{11}}{\lambda_{1}+i \sigma_{2}}\right)$ and $\Delta_{2}=\left(\frac{d_{11}}{\lambda_{1}-i \sigma_{2}}\right)$

The wall shear stress of the fluid at the lower plate is given by

$-\left(\frac{\partial u}{\partial y}\right)_{y=0}=a_{11}\left[\left(L_{1} \cos \sigma_{1} t-M_{1} \sin \sigma_{1} t\right)+i\left(M_{1} \cos \sigma_{1} t+L_{1} \sin \sigma_{1} t\right)\right]+\mathrm{b}_{11}\left[\left(L_{1} \cos \sigma_{1} t-M_{1} \sin \sigma_{1} t\right)-i\left(M_{1} \cos \sigma_{1} t\right.\right.$

$\left.\left.+L_{1} \sin \sigma_{1} t\right)\right]+c_{11}\left[\left(M_{2} \sin \sigma_{2} t-L_{2} \cos \sigma_{2} t\right)-i\left(M_{2} \sin \sigma_{2} t+L_{2} \sin \sigma_{2} t\right)\right]+\mathrm{d}_{11}\left[\left(M_{2} \sin \sigma_{2} t-L_{2} \cos \sigma_{2} t\right)+i\left(M_{2} \cos \sigma_{2} t\right.\right.$

$\left.\left.+L_{2} \sin \sigma_{2} t\right)\right]+2 \pi^{2} \sum_{n=1}^{\infty} n^{2}\left(\Delta_{1}+\Delta_{2}\right) e^{\lambda_{1} t}-2 \pi^{2} \sum_{n=1}^{\infty}(-1)^{n}\left(\Delta_{3}+\Delta_{4}\right) e^{\lambda_{1} t}$

Where

$L_{1}=\frac{1}{K_{3}}\left\{a_{2} \cos a_{2} \sin a_{2}+a_{1} \cosh a_{1} \sinh a_{1}\right\}$

$M_{1}=\frac{1}{K_{3}}\left\{a_{2} \sinh _{1} \cosh \mathrm{a}_{1}-a_{1} \cos \mathrm{a}_{2} \sin \mathrm{a}_{2}\right\}$

$L_{2}=\frac{1}{K_{3}^{\prime}}\left\{\mathrm{a}_{3} \mathrm{~K}_{1}^{\prime}+a_{4} \mathrm{~K}_{2}^{\prime}\right\}$

And $M_{2}=\frac{1}{K_{3}^{\prime}}\left\{\mathrm{a}_{4} \mathrm{~K}_{2}^{\prime}-a_{3} \mathrm{~K}_{1}^{\prime}\right\}$

Skin friction of the fluid is given by

$$
\begin{aligned}
& \left.\frac{\partial^{2} u}{\partial y^{2}}\right|_{y=0}=a_{11}\left\{\left(L_{2}^{\prime} \cos \sigma_{1} t-M_{2}^{\prime} \sin \sigma_{1} t\right)+i\left(M_{2}^{\prime} \cos \sigma_{1} t+L_{2}^{\prime} \sin \sigma_{1} t\right)\right\}+b_{11}\left\{\left(L_{2}^{\prime} \cos \sigma_{1} t-M_{2}^{\prime} \sin \sigma_{1} t\right)-i\left(M_{2}^{\prime} \cos \sigma_{1} t+L_{2}^{\prime} \sin \sigma_{1} t\right)\right\} \\
& L_{2}^{\prime}=\frac{1}{K_{3}}\left\{\left(l_{3}^{2} l_{2}^{2}+l_{4}^{2} l_{1}^{2}\right)\left(a_{1}^{2}-a_{2}^{2}\right)\right\} \\
& M_{2}^{\prime}=\frac{1}{K_{3}}\left(4 a_{1} a_{2} l_{3}^{2} l_{2}^{2}\right) \\
& l_{1}=\operatorname{sina}_{2} ; l_{2}=\operatorname{cosa}_{2} ; l_{3}=\operatorname{sinha}_{1} ; l_{4}=\operatorname{cosha}_{1}
\end{aligned}
$$

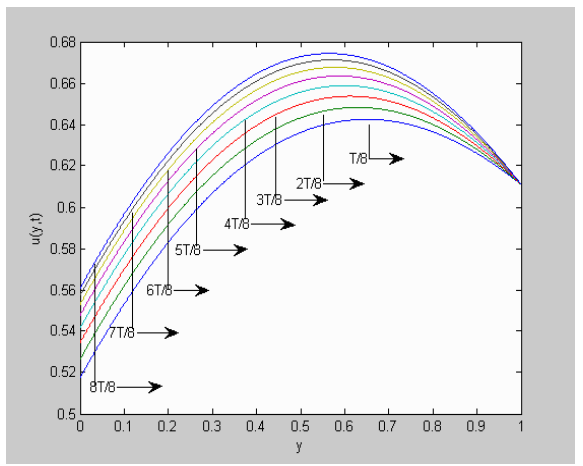

Figure 2: Velocity Sketch for $\mathbf{n = 0 . 5 , T = 1 . 0}$ with $\sigma=0.5$. 


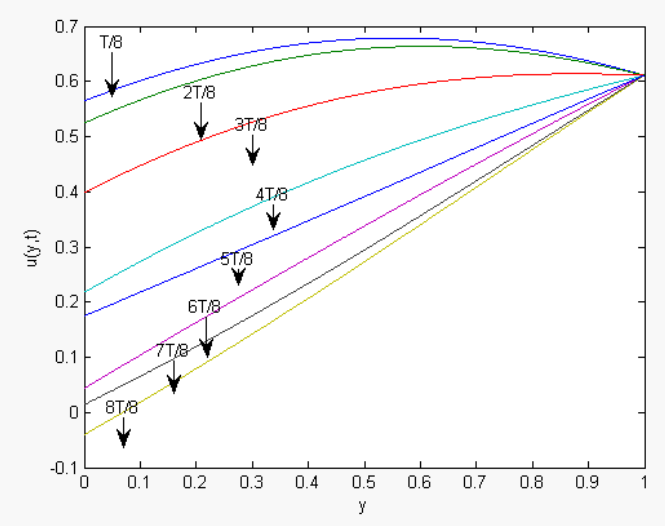

Figure 3: Velocity Sketch for $\mathbf{n = 0 . 5 ,} \mathbf{T}=10.0$ with $\sigma=0.5$.

Table 1: Wall Shear Stress of the Fluid

\begin{tabular}{|c|c|c|c|c|c|c|}
\hline $\mathbf{t}$ & \multicolumn{5}{|c|}{$T=1.0$} & \multicolumn{3}{c|}{$T=10.0$} \\
\hline$\frac{T}{8}$ & 5.3861 & 25.4942 & -51.6681 & $7.8458 \mathrm{e}+049$ & $1.9323 \mathrm{e}+003$ & 4.9970 \\
\hline$\frac{2 T}{8}$ & 3.4702 & -12.4946 & -92.2923 & $1.6238 \mathrm{e}+047$ & $1.6555 \mathrm{e}+003$ & 5.1512 \\
\hline$\frac{3 T}{8}$ & 1.2859 & $11.563-8$ & $2.7328 \mathrm{e}+040$ & $7.5579 \mathrm{e}+042$ & $1.2733 \mathrm{e}+003$ & 5.2810 \\
\hline$\frac{4 T}{8}$ & 0.1918 & 18.5155 & $2.9911 \mathrm{e}+017$ & $11223 \mathrm{e}+037$ & 885.2723 & 5.3844 \\
\hline$\frac{5 T}{8}$ & -0.2054 & $2.0033 \mathrm{e}+003$ & -193.9769 & $8.5102 \mathrm{e}+029$ & 562.5149 & 5.4596 \\
\hline$\frac{6 T}{8}$ & -0.2734 & 28.4464 & $-1.6760 \mathrm{e}+013$ & $5.8547 \mathrm{e}+021$ & 328.6581 & 5.5054 \\
\hline$\frac{7 T}{8}$ & -0.0397 & -12.2314 & $9.6287 \mathrm{e}+042$ & $7.0616 \mathrm{e}+012$ & 172.6419 & 5.5209 \\
\hline$\frac{8 T}{8}$ & 0.6579 & -10.5047 & -88.4185 & $3.1558 \mathrm{e}+003$ & 79.1751 & 5.5060 \\
\hline
\end{tabular}

Table 2: Skin Friction of the Fluid

\begin{tabular}{|c|c|c|c|c|c|c|}
\hline $\mathbf{t}$ & \multicolumn{3}{|c|}{$T=\mathbf{1 . 0}$} & \multicolumn{3}{c|}{ T=10.0 } \\
\hline$\frac{T}{8}$ & -0.2372 & -0.0278 & $1.1409 \mathrm{e}+003$ & -32.690 & -0.1462 & -0.4934 \\
\hline$\frac{2 T}{8}$ & -0.2666 & -0.0397 & 454.4805 & -58.9868 & -0.2037 & -0.6806 \\
\hline$\frac{3 T}{8}$ & -0.2963 & -0.0525 & -597.9239 & 253.7112 & -0.1167 & -0.7655 \\
\hline$\frac{4 T}{8}$ & -0.3260 & -0.0658 & -469.4783 & 373.4170 & 0.0031 & -0.7832 \\
\hline$\frac{5 T}{8}$ & -0.3556 & -0.0795 & 5.5980 & -847.8426 & -0.0143 & -0.7357 \\
\hline$\frac{6 T}{8}$ & -0.3847 & -0.0933 & 241.7083 & 928.1323 & -0.1430 & -0.6103 \\
\hline$\frac{7 T}{8}$ & -0.4133 & -0.1071 & -217.9592 & 725.1963 & -0.2041 & -0.3672 \\
\hline$\frac{8 T}{8}$ & -0.4410 & -0.1207 & 126.6171 & $-1.5292 \mathrm{e}+003$ & -0.1202 & -0.0913 \\
\hline
\end{tabular}




\section{RESULTS AND DISCUSSION}

In figure 1 , the velocity profile meant for the liquefied are shown for unusual values of $\mathrm{t}$, when $\mathrm{T}=1.0, \mathrm{n}=0.5, \sigma=0.5$.It is seen to the velocity increases while the time episode of the oscillation increases. But the reverse effect is also seen in figure 2 when $\mathrm{T}=1.0, \mathrm{n}=0.5, \sigma=0.5$. It is clear that frequencies are high for the smaller point age of oscillation of the plate. On the other hand, frequencies are low on behalf of the larger instant stage of the oscillation of the plate. From table 1and table 2, illustrate that the wall shear stress and skin friction of the solution for different values of t with three cases i) $\sigma<1$ ii) $\sigma=1$ iii) $\sigma>1$. The wall shear stress on the lesser plate be calculated numerically with different parameters, and it is revealed that gradual decreases presented when $\sigma<1, \mathrm{~T}=1.0$ and more oscillation for the leftover values of $\sigma$. But the converse inclination is obtained in support of the case, as made known in the table. Skin friction results are exposed in the table with different flow parameters.

\section{CONCLUSIONS}

In this article, we applied the analytical method Elzaki transform method for finding the velocity profile, wall shear stress and skin friction with different time periods oscillation parameter and the same hydromagnetic parameter, electrical conductivity parameter. The Elzaki transform could be used to solve mathematical problems without any alternative to a new frequency domain.

\section{REFERENCES}

1. L. Debnath, A.K. Ghosh, “On unsteady hydromagnetic flows of a dusty viscous fluid between two oscillatory plates,” Journal of Applied Mathematics and Simulation, Vol. 2, pp. 13 - 31, 1999.

2. K. Vajravelu, J. Rivera, "Hydromagnetic flow at an oscillating plate," International Journal of Non-linear Mechanics, Vol. 38, pp. $305-312,2003$.

3. N.C. Ghosh, B.C. Ghosh, L. Debnath, "The Hydromagnetic flow of a dusty visco-elastic fluid between two infinite parallel plates," Computers and Mathematics with Applications, Vol. 39, pp. 103 - 116, 2000.

4. Senapati, N., R. K. Dhal, and K. Ray. "Effect of chemical reaction on MHD free convection on Kuvshinshki fluid through porous medium in presence of heat radiation with constant heat and mass flux across moving plate." International Journal of Applied Mathematics \& Statistical Sciences 2.4 (2013): 45-52.

5. I. Khan, A. Khan, A. Farhad,M.Qasim,S.Sharidan, "Unsteady hydromagnetic rotating flow through an oscillating porous plate embedded in a porous medium, "Mathematical Problems in Engineering,Vol.2013,pp.1-8.

6. Arunkumar Ghosh, Sanjib Kumar Datta, Pulakesh Sen, "On Hydromagnetic flow of an oldroyed - B fluid between two oscillating plates," International Journal of Applied Computer Maths., Vol. 2, pp. 365 - 386, 2016.

7. Senapati, Nityananda, and Rajendra Kumar Dhal. "Effect of Slip Condition on Unsteady MHD Oscillatory Flow in a Channel Filled with Porous Medium with Heat Radiation and Mass Transfer." Int. J. Appl. Math. Stat. Sci 2.3 (2013): 11-20.

8. A.K. Ghosh, L. Debnath, "Hydromagnetic stokes flow in a rotating fluid with suspended small particles," Applied Scientific Research, Vol. 43, pp. 165 - 192, 1986.

9. P. Mitra, Bhattacharyya, "On the Hydromagnetic flow of a dusty fluid between two parallel plates," Journal of the Physical Society of Japan, Vol. 50, pp. 995 - 1001, 1981. 
10. Ali J. Chamkha, "Hydromagnetic combined convection flow in a vertical LID - Driven cavity with internal heat generation or absorption," Numerical Heat Transfer, Vol. 41, pp. 529 - 546, 2002.

11. Reddy, D. Babu, and G. S. S. Raju. "Hall Current Effects on MHD Free Convective Heat and Mass Transfer Flow Past an Oscillating Vertical Porous Plate in the Presence of Radiation, Thermal \& Mass Diffusion with Chemical Reaction." (2017). International Journal of Mathematics and Computer Applications Research (IJMCAR) 7. 2, Apr 2017, 1-22

12. Gauri Shankar Seth, Rohit Sharma, Bidyasugar Kumbhakar, Chamkha Ali J., "Hydromagnetic flow of heat absorbing and radiating fluid over exponentially stretching sheet with partial slip and viscous and Joule dissipation," Engineering Computations, Vol. 3, pp. 907 - 925, 1984.

13. S.P. Anjali Devi, T. Elakkiya Priya, "Numerical investigation of slip effects on Hydromagnetic flow due to a rotating disk in a nano fluid with internal heat absorption," Journal of Mechanics, Vol. 33, pp. 375 - 380, 2007.

14. Humbe, A. B., et al. "Review of laser plastic welding process." Int. J. Res. Eng. Technol 2 (2014): 191-206.

15. Sankar Kumar Guchhait, Rabindra Nath Jana, Sanatan das, "Hall effects on Hydromagnetic free convection in a heated vertical channel in the presence of an inclined magnetic field and thermal radiation," Turkish Journal of Engineering and Environmental Sciences, Vol. 38, pp. $434-454$.

16. G.C. Pande, G.A. Georgantopoulos, C.L. Goudas, "Hydromagnetic flow near an oscillating wall," Astrophysics and Space Science, Vol. 43, pp. $403-409,1976$.

17. A. Hazeem Attia, M. Kareem Ewis, "Hydromagnetic flow of a conducting - micropolar fluid over a plane wall with heat transfer," Tamkang Journal of Science and Engineering, Vol. 14, pp. 1 - 6, 2011.

18. M. Subhas Abel, G. Prashant Metri, "Hydromagnetic flow of a thin nanofluid film over an unsteady stretching sheet," International Journal of Advances in Applied Mathematics and Mechanics, Vol. 3, pp. 124 - 134, 2016.

19. Ramasamy Kandasamy, Karuppanan Periya Samy, "Non-linear Hydromagnetic flow, heat and mass transfer over an accelerating vertical surface with internal heat generation and thermal stratification effects,", Journal of Computational and Applied Mechanics, Vol. 6, pp. 27 - 37, 2005.

20. M.Veera Krishna,Kamboji Jyothi "Hall effects on MHD rotating flow of a visco elastic fluid through a porous medium over an infinite oscillating porous plate with heat source and chemical reaction, Materials today proceedings.Vol.5,pp.367-480,2018.

21. Sami Ullah Khan,Nasir Ali,Zaheer Abbas,Hydromagnetic "Flow and heat transfer over a porous oscillating stretching stretching surface in a viscoelastic fluid with porous medium"PlOS one, Vol.12,2015.

22. Ahmad Gushairi Mohamad, Iiyas Khan, Zulkhibri Ismail and Sharidan Shafiel, "Exact solutions for unsteady free convection flow over an oscillating plate due to non-coaxial rotation," Springer plus, Vol. 5, . doi: 10:1186/s40064-016-3748-2.

23. Iiyas Khan, Nehad Ali Shah, L.C.C. Dnnis, "A scientific report on heat transfer analysis in mixed convocation flow of Maxwell fluid over an oscillating vertical plate," Scientific Reports, Vol. 7, no. 40147, doi: 10.1038/srep40147.

24. Sahin Ahmed, Abdul Batin, "Hydromagnetic oscillatory convective flow through porous medium in a rotating vertical porous channel with thermal radiation effects," International Journal of Engineering Sciences and Research Technology, Vol. 3, 2014.

25. M. Veerakrishna, G. Sudha Reddy, A.J. Chamkha, "Hall effects on unsteady MHD oscillatory free convective flow of second grade fluid through porous medium between two vertical plates," Physics of Fluids, Vol. 30, no. 023106, 2018. http://doi.org/10/1063/1.5010863. 
26. S. Rashmi, V. Kavitha, B. Saha Roohi, Gurumurthy B.J. Gireesha and C.S. Bagewadi, "Unsteady flow of a dusty fluid between two oscillating plates under varying constant pressure gradient," NOVI SAD, J. Math., Vol. 37, pp. 25 - 34, 2007.

27. J.N. Tokis, V.S. Geroyannis, "Unsteady Hydromagnetic rotating flow near an oscillating plate," Astrophysics and Space Science, Vol. 75, pp. 393 - 405, 1981.

28. N. Abhas, Y. Wang, T. Hayat, M. Oberlack, "Hydromagnetic flow in a viscoelastic fluid due to the oscillatory stretching surface," International Journal of Non-linear Mechanics, doi: 10.1016/j.ijnlinmec.2008.04.009.

29. Mohamed ABD El-Aziz, S. Aishah, Yahya, "Heat and Mass transfer of unsteady Hydromagnetic free convection flow through porous medium past a vertical plate with uniform surface heat flux,", Journal of Theoretical and Applied Mechanics, Vol. 47, pp. 25 - 58, 2002. Doi: 10.1515/jtam-2017-0013.

\section{AUTHORS PROFILE}

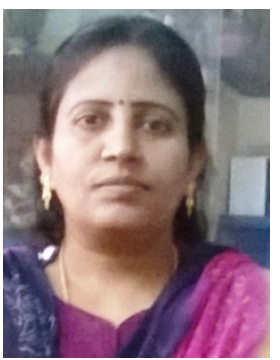

Dr. B. Vennila is from Perungalathur near Chennai. She is working as an Associate professor in SRM Institute of science and technology, Chennai. She received her Ph.D. Degree in the area of Fluid dynamics from SRM University Chennai in 2010.She completed her MPhil mathematics from Alagappa University, Karaikudi in the year 1996. B.sc and M.sc Mathematics from Seethalakshmi Achi College for women, Karaikudi in the year 1990-1995.Her area of research interest is rotating fluid and streetching sheet problems. She is a member of ISTE, IMS, IAENG and ISCO.

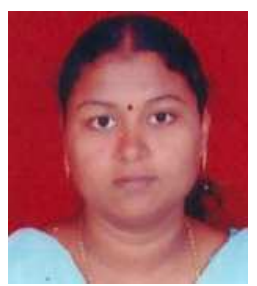

Mrs. K. Suganthi is from Peungalathur near Chennai. She is working as an Assistant Professor in SRM University, Chennai. Currently, pursuing her research in RF VLSI in the same University. She completed her M.Tech. VLSI Design from SRM University, Chennai and B.E. Electronics and Communication from Madras University in the year 2007 and 2003 respectively. Her research interests are in the area of VLSI Design, Micro-electronics, RF VLSI, She is a member of ISTE and IEEE. 